\title{
Suffix Array of Alignment: A Practical Index for Similar Data
}

\author{
Joong Chae $\mathrm{Na}^{1}$, Heejin Park ${ }^{2}$, Sunho Lee ${ }^{3}$, Minsung Hong ${ }^{3}$, \\ Thierry Lecroq ${ }^{4}$, Laurent Mouchard ${ }^{4}$, and Kunsoo Park ${ }^{3, \star}$ \\ 1 Department of Computer Science and Engineering, Sejong University, Korea \\ jcna@sejong.ac.kr \\ 2 College of Information and Communications, Hanyang University, Korea \\ hjpark@hanyang.ac. kr \\ 3 School of Computer Science and Engineering, Seoul National University, Korea \\ \{slee,mshong, kpark\}@theory.snu.ac.kr \\ 4 Department of Computer Science, University of Rouen, France \\ $\{$ Thierry.Lecroq, Laurent.Mouchard\}@univ-rouen.fr
}

\begin{abstract}
The suffix tree of alignment is an index data structure for similar strings. Given an alignment of similar strings, it stores all suffixes of the alignment, called alignment-suffixes. An alignment-suffix represents one suffix of a string or suffixes of multiple strings starting at the same position in the alignment. The suffix tree of alignment makes good use of similarity in strings theoretically. However, suffix trees are not widely used in biological applications because of their huge space requirements, and instead suffix arrays are used in practice.

In this paper we propose a space-economical version of the suffix tree of alignment, named the suffix array of alignment (SAA). Given an alignment $\rho$ of similar strings, the SAA for $\rho$ is a lexicographically sorted list of all the alignment-suffixes of $\rho$. The SAA supports pattern search as efficiently as the generalized suffix array. Our experiments show that our index uses only $14 \%$ of the space used by the generalized suffix array to index 11 human genome sequences. The space efficiency of our index increases as the number of the genome sequences increases. We also present an efficient algorithm for constructing the SAA.
\end{abstract}

Keywords: Indexes for similar data, suffix arrays, alignments

\section{Introduction}

The 1000 Genomes project [4] is aiming at building a database of 1092 individual human genome sequences using a cheap and fast sequencing, called Next Generation Sequencing (NGS). To sequence an individual genome using the NGS, the individual genome is divided into short segments (called reads) and they are aligned to the Human reference Genome. This is possible because an individual

\footnotetext{
${ }^{\star}$ Corresponding author.
} 
genome is more than $99 \%$ identical to the Human reference Genome. The similarity also enables us to store individual genomes efficiently. Instead of storing 1000 whole individual sequences, only $1 \%$ different regions of each individual genome can be stored.

Not only efficient storing techniques but also efficient indexing techniques for similar strings have been developed. The first such index was proposed by Mäkinen et al. $[16,17]$. Their index uses run-length encoding, a suffix array, and BWT [3]. Huang et al. [9] indexed similar strings by building separate data structures for common regions and non-common regions. In addition, indexes based on Lemple-Ziv compression schemes [15, 21] have been developed $[5,14]$. Some of these indexes are surveyed in [20]. The space reductions of these indexes are achieved mostly by using classical compressed indexes. However, the indexes do not support efficient pattern search or require auxiliary data structures to improve the pattern search time.

Recently, a suffix tree for similar strings, called a suffix tree of alignment [19], have been proposed without sacrificing the pattern search time, i.e., the suffix tree of alignment supports linear-time pattern search. Given an alignment of similar strings, the suffix tree of alignment stores suffixes of an alignment, called alignment-suffixes (for short a-suffixes) rather than suffixes of a string. An asuffix may represent suffixes of multiple strings starting at the same position in an alignment. The suffix tree of alignment makes good use of similarity in strings theoretically. Although suffix trees support many functionalities $[2,8]$, however, they are not widely used in biological applications because of the huge space requirement. Instead, suffix arrays [18] (including their compressed forms [6,7]) are widely used in practice.

In this paper we propose the suffix array of alignment (SAA), an array version of the suffix tree of alignment. Given an alignment $\rho$, the SAA for $\rho$ is a lexicographically sorted list of all the a-suffixes of $\rho$. We show that the sorted order of the a-suffixes is well defined and the longest common prefix (lcp) of two a-suffixes is also well defined. Assume that given strings consist of common regions and non-common regions alternatively, e.g., three strings $A, B$, and $C$ can be represented as $A=\alpha_{1} \beta_{1} \ldots \alpha_{k} \beta_{k} \alpha_{k+1}, B=\alpha_{1} \delta_{1} \ldots \alpha_{k} \delta_{k} \alpha_{k+1}$, and $C=\alpha_{1} \vartheta_{1} \ldots \alpha_{k} \vartheta_{k} \alpha_{k+1}$, where $\alpha_{i}$ 's are common regions and $\beta_{i}$ 's, $\delta_{i}$ 's and $\vartheta_{i}$ 's are non-common regions. Then, the SAA requires $O\left(|A|+\sum_{i=1}^{k}\left(2\left|\alpha_{i}^{*}\right|+\left|\delta_{i}\right|+\left|\vartheta_{i}\right|\right)\right)$ space, where $\alpha_{i}^{*}$ is the longest suffix of $\alpha_{i}$ appearing at least twice in $A$, in $B$ or in $C$. (For simplicity, three strings are considered but our results work well for more than three strings.) The space requirement of the SAA is asymptotically the same as that of the suffix tree of alignment, but the SAA is more space-efficient practically. Furthermore, our suffix array supports pattern search as efficiently as the generalized suffix array (GSA).

Moreover, we show by experiments that our index is space-efficient for similar data in practice by analyzing and comparing the space requirements of the SAA and the GSA, which support the same efficiency of pattern search. The space requirement of our index is influenced by the lengths of $\alpha_{i}^{*}$ and non-common regions. Our experiments show that these lengths are short in practice and thus 
our index consumes very small space. We used 11 human genome sequences, one reference sequence and 10 individual sequences from the 1000 Genomes project website $^{5}$. In the genome sequences, non-common regions are only $0.3 \%$ of the entire positions, i.e., these sequences are very similar. Moreover, the $\alpha_{i}^{*}$ 's, which is a main factor for the space requirement of the SAA, occupy $5 \%$ of the entire positions and the length of $\alpha_{i}^{*}$ is 16.64 on average. Conclusively, the SAA requires only $14 \%$ of the space required by the GSA for indexing the 11 sequences. It should be noted that the space efficiency of our index increases as the number of the genome sequences increases.

We also present an efficient algorithm for constructing the SAA. One might think the SAA can be simply constructed by simulating the algorithm for constructing the suffix tree of alignment in [19]. However, it is not easy because the algorithm heavily uses the dynamic property of the suffix tree and makes use of suffix links. The core of the tree construction algorithm is how to compute $\alpha_{i}^{*}$ efficiently, which is solved using a property satisfied in a partial suffix tree containing suffixes derived from several strings. Thus, we developed a new algorithm to compute $\alpha_{i}^{*}$ using only suffix arrays. For this, we generalize the property dedicated to the suffix tree so that the property is satisfied in substrings of input strings. Conclusively, we can compute $\alpha_{i}^{*}$ and thus construct the SAA as efficiently as the algorithm in [19].

\section{Suffix array of alignment (SAA)}

In this section we define the suffix array of alignment (SAA) and present how to construct the SAA. For simplicity, we consider only alignments of three strings but our definitions and algorithms can be easily extended to more than three strings. We first consider alignments with one non-common chunk and then general alignments with more than one non-common chunk.

\subsection{Definition of SAA}

Let $A, B$, and $C$ be similar strings such that $A=\alpha \beta \gamma, B=\alpha \delta \gamma$, and $C=\alpha \vartheta \gamma$, where $\alpha$ and $\gamma$ are common regions in all strings, and $\beta, \delta$, and $\vartheta$ are non-common regions. Then, these regions represent an alignment of the strings and each string can be transformed to another string by replacing non-common regions. We denote this alignment of the three strings by $\rho=\alpha(\beta / \delta / \vartheta) \gamma$. For simplicity, we assume that all strings end with a special symbol $\# \in \Sigma$ occurring nowhere else in the strings.

The suffixes of the alignment $\rho$, called alignment-suffixes (for short a-suffixes), are defined as in [19]. Let $\alpha^{a}, \alpha^{b}$, and $\alpha^{c}$ be the longest suffixes of $\alpha$ occurring at least twice in the strings $A, B$, and $C$, respectively. Let $\alpha^{*}$ be the longest of $\alpha^{a}, \alpha^{b}$, and $\alpha^{c}$, i.e., $\alpha^{*}$ is the longest suffix of $\alpha$ occurring at least twice in $A$, in $B$, or in $C$. Then, these are a-suffixes of $\rho$, which are classified into 5 types.

\footnotetext{
${ }^{5}$ http://www.1000genomes.org/
} 


\begin{tabular}{|c|c|c|c|}
\hline$i d x$ & $P O S$ & $L C P$ & a-suffixes (type) \\
\hline 1 & $(1,9)$ & - & $\#(1)$ \\
\hline 2 & $(2,6)$ & 0 & a a b \# (3) \\
\hline 3 & $(1,4)$ & 3 & $\mathrm{a} \underline{\mathrm{ab}} \mathrm{ab} \#(2)$ \\
\hline 4 & $(3,4)$ & 2 & $\mathrm{a} \underline{\mathrm{ac}} \mathrm{ab} \#(4)$ \\
\hline 5 & $(1,7)$ & 1 & $\mathrm{a} b \#(1)$ \\
\hline 6 & $(2,4)$ & 2 & $\mathrm{a} \underline{\mathrm{b} a} \mathrm{ab} \#(3)$ \\
\hline 7 & $(1,5)$ & 3 & $\underline{a b} a b \#(2)$ \\
\hline 8 & $(1,1)$ & 2 & $\mathrm{abca}(\mathrm{ab} / \mathrm{ba} / \mathrm{ac}) \mathrm{ab} \#(5)$ \\
\hline 9 & $(3,5)$ & 1 & 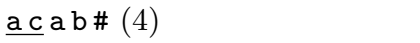 \\
\hline$\vdots$ & & $\vdots$ & $\vdots$ \\
\hline
\end{tabular}

Fig. 1. The SAA of abca (ab/ba/ac) ab\#. A pair $(a, b)$ in $P O S$ represents the string number $a$ and the starting position $b$ of an a-suffix. $L C P[i]$ is the length of lcp between two a-suffixes of $P O S[i-1]$ and $P O S[i]$.

1. a suffix of $\gamma$,

2. $\omega^{a} \gamma$, where $\omega^{a}$ is a (non-empty) suffix of $\alpha^{*} \beta$.

3. $\omega^{b} \gamma$, where $\omega^{b}$ is a (non-empty) suffix of $\alpha^{*} \delta$.

4. $\omega^{c} \gamma$, where $\omega^{c}$ is a (non-empty) suffix of $\alpha^{*} \vartheta$.

5. $\alpha^{\prime}(\beta / \delta / \vartheta) \gamma$, where $\alpha^{\prime}$ is a suffix of $\alpha$ longer than $\alpha^{*}$.

For example, assume that an alignment $a b c a(a b / b a / a c) a b \#$ is given. Then, $\alpha^{a}=\alpha^{b}=\mathrm{a}$ and $\alpha^{c}=\mathrm{ca}$. Since $\alpha^{*}$ is ca, caabab\# is an a-suffix of type 2 and $\mathrm{bca}(\mathrm{ab} / \mathrm{ba} / \mathrm{ac}) \mathrm{ab \#}$ is an a-suffix of type 5 .

The suffix array of alignment (SAA) for $\rho$ is a lexicographically sorted list of all the a-suffixes of $\rho$. It is clear what the sorted order for a-suffixes of types 1-4 is since an a-suffix of types 1-4 represents one string. On the other hand, an a-suffix of type 5, e.g., $\omega=\alpha^{\prime}(\beta / \delta / \vartheta) \gamma$ where $\left|\alpha^{\prime}\right|>\left|\alpha^{*}\right|$ represents three strings $\alpha^{\prime} \beta \gamma$, $\alpha^{\prime} \delta \gamma$, and $\alpha^{\prime} \vartheta \gamma$ derived from $A, B$, and $C$, respectively. However, it does not cause trouble when determining the order of $\omega$ between the a-suffixes of $\rho$. Since $\alpha^{\prime}$ occurs only once in each string, i.e, as prefix of $\alpha^{\prime} \beta \gamma, \alpha^{\prime} \delta \gamma$, and $\alpha^{\prime} \vartheta \gamma$, the order of $\omega$ is determined by $\alpha^{\prime}$. Thus, the lexicographically sorted order between the a-suffixes is well defined and the longest common prefix (lcp), an additional information often used together with the suffix arrays, between a-suffixes of $\rho$ is also well defined. See Figure 1 for an example.

The space requirement of the SAA is linear to the number of a-suffixes. There are $|\gamma|$ a-suffixes of type 1 . The number of a-suffixes of types 2,3 , and 4 is $\left.\left|\alpha^{*} \beta\right|+\left|\alpha^{*} \delta\right|+\left|\alpha^{*} \vartheta\right|\right)$ and the number of a-suffixes of type 5 is $|\alpha|-\left|\alpha^{*}\right|$. Since $|A|=|\alpha|+|\beta|+|\gamma|$, the SAA of $\rho$ requires $O\left(|A|+2\left|\alpha^{*}\right|+|\delta|+|\vartheta|\right)$ space.

\subsection{Construction of SAA}

One method for constructing the SAA of $\rho$ is using the suffix tree of the alignment $\rho[19]$ as an intermediate index. However, this method does not make full use of the space-efficiency of suffix arrays because suffix trees require much more 
space than suffix arrays. Another method, without constructing suffix trees, is constructing first the generalized suffix array (GSA) for the three strings as an intermediate index and then deleting suffixes that are not a-suffixes in the GSA. However, this method also is not efficient in working space as well as in construction time because the time and space requirement of the GSA is proportional to the total length of the strings regardless of similarity among the strings. The more number of strings are in the alignment and the more similar the strings are, the more is the inefficiency.

We present how to construct the SAA efficiently in time and space. Our algorithm for constructing the SAA of $\rho$ consists of three steps. Let $\gamma^{a}, \gamma^{b}$, and $\gamma^{c}$ be the longest prefixes of $\gamma$ occurring at least twice in the strings $A, B$, and $C$, respectively. Let $\gamma^{*}$ be the longest of $\gamma^{a}, \gamma^{b}$, and $\gamma^{c}$. (Note that these definitions are symmetrical with those of $\alpha^{a}, \alpha^{b}, \alpha^{c}$, and $\alpha^{*}$, and are different from the definition of $\hat{\gamma}$ used in [19].) Then, the outline of our algorithm is as follows:

1. Compute $\left|\alpha^{*}\right|$ and $\left|\gamma^{*}\right|$.

2. Construct the GSA for three strings $A, \alpha^{*} \delta \gamma^{*} d$, and $\alpha^{*} \vartheta \gamma^{*} d$, where $d$ is the symbol following $\gamma^{*}$ in $\gamma$.

3. Delete suffixes of $\gamma^{*} d$ derived from $\alpha^{*} \delta \gamma^{*} d$ and $\alpha^{*} \vartheta \gamma^{*} d$.

Step 1 is the core step of our algorithm. We mainly focus on the problem of computing $\left|\alpha^{*}\right|$ since $\left|\gamma^{*}\right|$ can be computed symmetrically. For a string $S$, let $S^{R}$ be the reversed string of $S$. We can compute $\left|\alpha^{a}\right|$ by searching for $\alpha^{R}$ in the suffix array of $A^{R}$. Thus, one method to compute $\left|\alpha^{*}\right|$ is constructing the suffix array of each reversed string and computing $\left|\alpha^{a}\right|,\left|\alpha^{b}\right|$, and $\left|\alpha^{c}\right|$. However, this method requires the time proportional to the total length of the three strings due to constructing the three suffix arrays.

To compute $\left|\alpha^{*}\right|$ more efficiently, we make use of the similarity in the strings. Consider the strings $A$ and $B$. The following lemma says that, given $\left|\alpha^{a}\right|$, a substring including $\delta$ is sufficient for computing $\max \left(\left|\alpha^{a}\right|,\left|\alpha^{b}\right|\right)$ instead of the entire of $B$. (Note that we do not need to compute the exact value of $\left|\alpha^{b}\right|$ to compute $\left|\alpha^{*}\right|$.)

Lemma 1. If $\left|\alpha^{b}\right|>\left|\alpha^{a}\right|, \alpha^{b}$ occurs in the substring $B^{\prime}$ of $B$, where $B^{\prime}=\alpha^{a} \delta \gamma^{a}$.

Proof. By definition of $\alpha^{b}$, there are at least two occurrences of $\alpha^{b}$ in $B$. Obviously, one occurrence occ $_{1}$ of $\alpha^{b}$ appears as a suffix of $\alpha$. Since $\left|\alpha^{b}\right|>\left|\alpha^{a}\right|$, $o c c_{1}$ cannot be included in $B^{\prime}$. Let $o c c_{2}$ denote an occurrence of $\alpha^{b}$ in $B$ other than $o c c_{1}$. Let $s_{2}$ and $e_{2}$ be the starting and the ending positions of $o c c_{2}$ in $B$, respectively. Let $s_{a}$ and $e_{a}$ be the starting and the ending positions of the substring $B^{\prime}$ in $B$, respectively.

We show that $o c c_{2}$ is included in $B^{\prime}$, i.e., $s_{a} \leq s_{2}$ and $e_{2} \leq e_{a}$. We first prove by contradiction that $s_{a} \leq s_{2}$. Suppose $s_{2}<s_{a}$. We have two cases according to whether $o c c_{2}$ is overlapped with $\delta$ or not.

- The case when $o c c_{2}$ is not overlapped with $\delta$. Then, $o c c_{2}$ is included in $\alpha$ and it means that there are at least two occurrences $\left(o c c_{1}\right.$ and $\left.o c c_{2}\right)$ of $\alpha^{b}$ in $\alpha$ and also in $A$. It contradicts with the definition of $\alpha^{a}$ since $\left|\alpha^{b}\right|>\left|\alpha^{a}\right|$. 
- The case when $o c c_{2}$ is overlapped with $\delta$. Let $\alpha^{\prime}$ be the suffix of $\alpha$ starting at $s_{2}$. Since $s_{2}<s_{a},\left|\alpha^{\prime}\right|>\left|\alpha^{a}\right|$. Since $\alpha^{\prime}$ is a prefix of $o c c_{2}, \alpha^{\prime}$ is also a prefix of $o c c_{1}$. Hence, there are at least two occurrences of $\alpha^{\prime}$ in $\alpha$ and also in $A$. It contradicts with the definition of $\alpha^{a}$ since $\left|\alpha^{\prime}\right|>\left|\alpha^{a}\right|$.

Similarly, we can prove that $e_{2} \leq e_{a}$ by contradiction with the definition of $\gamma^{a}$.

Note that this property also holds for other strings. For example, if $\left|\alpha^{c}\right|>\left|\alpha^{a}\right|$, $\alpha^{c}$ occurs in the substring $\alpha^{a} \vartheta \gamma^{a}$ of $C$.

Using this property, we can compute $\left|\alpha^{*}\right|$ and $\left|\gamma^{*}\right|$ as follows:

1.1 Compute $\left|\alpha^{a}\right|$ by searching for $\alpha^{R}$ in the suffix array of $A^{R}$ and, symmetrically, compute $\left|\gamma^{a}\right|$ by searching for $\gamma$ in the suffix array of $A$.

1.2 Compute $\ell^{b}=\max \left(\left|\alpha^{a}\right|,\left|\alpha^{b}\right|\right)$ using the suffix array of $\left(\alpha^{a} \delta \gamma^{a}\right)^{R}$ as follows. Let $\alpha^{\prime}$ be the longest suffix of $\alpha$ occurring in $\alpha^{a} \delta \gamma^{a}$. (Note that $\left|\alpha^{\prime}\right| \geq\left|\alpha^{a}\right|$ since $\alpha^{a}$ occurs in $\alpha^{a} \delta \gamma^{a}$.) We can find $\alpha^{\prime}$ by searching for $\alpha^{R}$ in the suffix array of $\left(\alpha^{a} \delta \gamma^{a}\right)^{R}$. By Lemma 1, if $\left|\alpha^{\prime}\right|>\left|\alpha^{a}\right|, \ell^{b}=\left|\alpha^{\prime}\right|$ and, otherwise, $\ell^{b}=\left|\alpha^{a}\right|$.

Symmetrically, compute $\max \left(\left|\gamma^{a}\right|,\left|\gamma^{b}\right|\right)$ using the suffix array of $\alpha^{a} \delta \gamma^{a}$.

1.3 Similarly, compute $\ell^{c}=\max \left(\left|\alpha^{a}\right|,\left|\alpha^{c}\right|\right)$ using the suffix array of $\left(\alpha^{a} \vartheta \gamma^{a}\right)^{R}$. Then, $\left|\alpha^{*}\right|=\max \left(\ell^{b}, \ell^{c}\right)$.

Symmetrically, compute $\max \left(\left|\gamma^{a}\right|,\left|\gamma^{c}\right|\right)$ and $\left|\gamma^{*}\right|$.

Since the suffix array of a string $S$ can be constructed using $O(|S|)$ time and $O(|S|)$ space $[10,12,13]$, and one can search for a string $P$ using the suffix array of $S$ with some auxiliary information in $O(|P|)[1,11]$, computing $\left|\alpha^{*}\right|$ and $\left|\gamma^{*}\right|$ requires $O\left(|A|+\left|\alpha^{*} \delta \gamma^{*}\right|+\left|\alpha^{*} \vartheta \gamma^{*}\right|\right)$ time and $O(|A|)$ working space. Note that the suffix array constructed in each substep is needed only in the substep.

In Step 2, we construct the GSA for three strings $A, \alpha^{*} \delta \gamma^{*} d$, and $\alpha^{*} \vartheta \gamma^{*} d$, where $d$ is the symbol following $\gamma^{*}$ in $\gamma$. The GSA contains all the a-suffixes of the alignment $\rho$. (Note that the suffixes of $\gamma$ in $A$ are the a-suffixes of type 1 of $\rho$ and the suffixes of $A$ longer than $\alpha^{*} \beta \gamma$ can be implicitly converted to the a-suffixes of type 5 of $\rho$ [19].) The reason why $\gamma^{*} d$ is necessary is as follows. Let $\omega \gamma$ be an a-suffix of type 3 (of $B$ ). To determine the order of $\omega \gamma$ among a-suffixes of $\rho$, we may need a prefix of $\gamma$. Since $\gamma^{*} d$ occurs only once in each string, the order of $\omega \gamma$ is determined by $\omega \gamma^{*} d$. Obviously, Step 2 requires $O\left(|A|+\left|\alpha^{*} \delta \gamma^{*}\right|+\left|\alpha^{*} \vartheta \gamma^{*}\right|\right)$ time and space.

In Step 3, we delete suffixes of $\gamma^{*} d$ in $\alpha^{*} \delta \gamma^{*} d$ and $\alpha^{*} \vartheta \gamma^{*} d$ because these are redundant with suffixes of $A$ (a-suffixes of type 1). Consider a suffix $\omega$ of $\gamma^{*} d$. In the GSA, there are two $\omega$ 's derived from $\alpha^{*} \delta \gamma^{*} d$ and $\alpha^{*} \vartheta \gamma^{*} d$. The two $\omega$ 's are adjacent in the GSA. We can delete redundant suffixes by scanning the entire GSA, which requires $O\left(|A|+\left|\alpha^{*} \delta \gamma^{*}\right|+\left|\alpha^{*} \vartheta \gamma^{*}\right|\right)$ time and space.

Theorem 1. Given an alignment $\rho=\alpha(\beta / \delta / \vartheta) \gamma$, the SAA of $\rho$ can be constructed using $O\left(|A|+\left|\alpha^{*} \delta \gamma^{*}\right|+\left|\alpha^{*} \vartheta \gamma^{*}\right|\right)$ time and working space. 


\subsection{Alignment with multiple regions}

In this section we consider alignments with multiple non-common regions. Let $A=\alpha_{1} \beta_{1} \ldots \alpha_{k} \beta_{k} \alpha_{k+1}, B=\alpha_{1} \delta_{1} \ldots \alpha_{k} \delta_{k} \alpha_{k+1}$, and $C=\alpha_{1} \vartheta_{1} \ldots \alpha_{k} \vartheta_{k} \alpha_{k+1}$. We denote the alignment of the strings by $\rho=\alpha_{1}\left(\beta_{1} / \delta_{1} / \vartheta_{1}\right) \alpha_{2}\left(\beta_{2} / \delta_{2} / \vartheta_{2}\right) \alpha_{3} \ldots \alpha_{k+1}$. Without loss of generality, we assume that $\alpha_{i}(2 \leq i \leq k)$ occurs only once in each string. (Otherwise, we merge $\alpha_{i}$ with adjacent non-common regions, e.g., $\beta_{i-1} \alpha_{i} \beta_{i}$ is regarded as one non-common region). For $1 \leq i \leq k$, let $\alpha_{i}^{a}$, $\alpha_{i}^{b}$, and $\alpha_{i}^{c}$ be the longest suffixes of $\alpha_{i}$ occurring at least twice in the strings $A, B$, and $C$, respectively. Let $\alpha_{i}^{*}$ be the longest of $\alpha_{i}^{a}, \alpha_{i}^{b}$, and $\alpha_{i}^{c}$. Then, these are a-suffixes of $\rho$, which are classified into 5 types $(1 \leq i \leq k)$.

1. a suffix of $\alpha_{k+1}$,

2. $\omega_{i}^{a} \alpha_{i+1} \ldots \alpha_{k+1}$ where $\omega_{i}^{a}$ is a (non-empty) suffix of $\alpha_{i}^{*} \beta_{i}$.

3. $\omega_{i}^{b} \alpha_{i+1} \ldots \alpha_{k+1}$ where $\omega_{i}^{b}$ is a (non-empty) suffix of $\alpha_{i}^{*} \delta_{i}$.

4. $\omega_{i}^{c} \alpha_{i+1} \ldots \alpha_{k+1}$ where $\omega_{i}^{c}$ is a (non-empty) suffix of $\alpha_{i}^{*} \vartheta_{i}$.

5. $\alpha_{i}^{\prime}\left(\beta_{i} / \delta_{i} / \vartheta_{i}\right) \alpha_{i+1} \ldots \alpha_{k+1}$, where $\alpha_{i}^{\prime}$ is a suffix of $\alpha_{i}$ longer than $\alpha_{i}^{*}$.

The SAA of $\rho$ is a lexicographically sorted list of all the a-suffixes of $\rho$. The SAA requires the space linear to the number of a-suffixes, i.e., $O\left(|A|+\sum_{i=1}^{k}\left(2\left|\alpha_{i}^{*}\right|+\right.\right.$ $\left.\left.\left|\delta_{i}\right|+\left|\vartheta_{i}\right|\right)\right)$ space.

For $1 \leq i \leq k$, let $\gamma_{i}^{a}, \gamma_{i}^{b}$, and $\gamma_{i}^{c}$ be the longest prefix of $\alpha_{i+1}$ occurring at least twice in the strings $A, B$, and $C$, respectively, and let $\gamma_{i}^{*}$ be the longest of $\gamma_{i}^{a}, \gamma_{i}^{b}$, and $\gamma_{i}^{c}$. Let $B^{\prime}$ be the concatenation of the $k$ strings $\alpha_{i}^{a} \delta_{i} \gamma_{i}^{a} \#_{i}(1 \leq i \leq k)$ and $C^{\prime}$ be the concatenation of the $k$ strings $\alpha_{i}^{a} \vartheta_{i} \gamma_{i}^{a} \#_{i}(1 \leq i \leq k)$, where $\#_{i}$ is a delimiter. That is,

$$
\begin{gathered}
B^{\prime}=\alpha_{1}^{a} \delta_{1} \gamma_{1}^{a} \#_{1} \alpha_{2}^{a} \delta_{2} \gamma_{2}^{a} \#_{2} \ldots \alpha_{k}^{a} \delta_{k} \gamma_{k}^{a} \#_{k} \text { and } \\
C^{\prime}=\alpha_{1}^{a} \vartheta_{1} \gamma_{1}^{a} \#_{1} \alpha_{2}^{a} \vartheta_{2} \gamma_{2}^{a} \#_{2} \ldots \alpha_{k}^{a} \vartheta_{k} \gamma_{k}^{a} \#_{k} .
\end{gathered}
$$

Then, Lemma 1 can be generalized to the following lemma (we omit the proof).

Lemma 2. For every $i=1, \ldots, k$, if $\left|\alpha_{i}^{b}\right|>\left|\alpha_{i}^{a}\right|, \alpha_{i}^{b}$ occurs in $B^{\prime}$.

The SAA of $\rho$ can be constructed as follows:

1. Compute $\left|\alpha_{i}^{*}\right|$ and $\left|\gamma_{i}^{*}\right|(1 \leq i \leq k)$.

1.1 Compute $\left|\alpha_{i}^{a}\right|$, for every $i=1, \ldots, k$, by searching for $\alpha_{i}^{R}$ in the suffix array of $A^{R}$ and, symmetrically, compute $\left|\gamma_{i}^{a}\right|$ by searching for $\gamma_{i}$ in the suffix array of $A$.

1.2 Compute $\ell_{i}^{b}=\max \left(\left|\alpha_{i}^{a}\right|,\left|\alpha_{i}^{b}\right|\right)$ using the suffix array of $\left(B^{\prime}\right)^{R}$ as follows. Let $\alpha_{i}^{\prime}$ be the longest suffix of $\alpha_{i}$ occurring in $B^{\prime}$. We can find $\alpha_{i}^{\prime}$ by searching for $\left(\alpha_{i}\right)^{R}$ in the suffix array of $\left(B^{\prime}\right)^{R}$. By Lemma 2, if $\left|\alpha_{i}^{\prime}\right|>$ $\left|\alpha_{i}^{a}\right|, \ell_{i}^{b}=\left|\alpha_{i}^{\prime}\right|$ and, otherwise, $\ell_{i}^{b}=\left|\alpha_{i}^{a}\right|$.

Symmetrically, compute $\max \left(\left|\gamma_{i}^{a}\right|,\left|\gamma_{i}^{b}\right|\right)$ using the suffix array of $B^{\prime}$.

1.3 Similarly, compute $\ell_{i}^{c}=\max \left(\left|\alpha_{i}^{a}\right|,\left|\alpha_{i}^{c}\right|\right)$ using the suffix array of $\left(C^{\prime}\right)^{R}$. Then, $\left|\alpha_{i}^{*}\right|=\max \left(\ell_{i}^{b}, \ell_{i}^{c}\right)$.

Symmetrically, compute $\max \left(\left|\gamma_{i}^{a}\right|,\left|\gamma_{i}^{c}\right|\right)$ and $\left|\gamma_{i}^{*}\right|$.

2. Construct the GSA for $2 k+1$ strings $A, \alpha_{i}^{*} \delta_{i} \gamma_{i}^{*} d_{i}$, and $\alpha_{i}^{*} \vartheta_{i} \gamma_{i}^{*} d_{i}$, where $d_{i}$ is the symbol following $\gamma_{i}^{*}$ in $\alpha_{i+1}(1 \leq i \leq k)$.

3. Delete suffixes of $\gamma_{i}^{*} d_{i}$ derived from $\alpha_{i}^{*} \delta_{i} \gamma_{i}^{*} d_{i}$ and $\alpha_{i}^{*} \vartheta_{i} \gamma_{i}^{*} d_{i}(1 \leq i \leq k)$ in the GSA. 


\subsection{Pattern search}

We can perform pattern search using the SAA in the same way as using classical suffix arrays of strings except for dealing with alignments in a-suffixes. Consider an a-suffix $\omega\left(\beta_{i} / \delta_{i} / \vartheta_{i}\right) \ldots \alpha_{k+1}$ where $\omega$ does not contain an alignment. We can perform binary search with lcp information like in classical suffix arrays until a prefix of a given pattern $P$ matches $\omega$. If a prefix of $P$ matches $\omega$, we consider only the a-suffix to search for $P$ since $\omega$ occurs only once in each string by definition of a-suffixes. Thus, after a prefix of $P$ matches $\omega$, we compare $P$ with $\beta_{i}, \delta_{i}$, and $\vartheta_{i}$. We can enhance this comparison using the trie of $\beta_{i}, \delta_{i}$, and $\vartheta_{i}$.

\section{$3 \quad$ Experiments}

We show by experiments that our index (the SAA) is an effective data structure for similar data. The SAA requires only about $1 / 7$ of the space required by the GSA to index 11 human genome sequences, which is explained in the following.

\subsection{Experimental data}

To measure the space requirement of indexes in practice, we used one reference sequence and 10 individual sequences from 1000 Genomes project website. From the project website, we downloaded pairs of bam and bai files of 10 individual human genomes, where bam files contain reads (short segments of length 90125) of each individual and bai files contain alignment of the reads. We also downloaded their corresponding reference genome, hg19. To convert a set of reads into one sequence, we used samtools ${ }^{6}$ (Sequence Alignment/Map tools), by which we obtained 10 individual genome sequences. Since individual genome sequences are aligned to the reference genome sequence, these 11 sequences make a multiple alignment based on the reference genome sequence.

In our experiments, we used chromosome 20 of each genome. The length of the reference sequence is $63,025,520$ and the lengths of the individual sequences vary from $62,965,442$ to $62,965,512$, which are a little shorter than the reference sequence. The sequences consist of five characters $\{A, G, T, C, N\}$, where $A, G$, $T$, and $C$ stand for nucleotides Adenine, Guanine, Thymine, and Cytosine, respectively, and $N$ appears in some special cases and is treated exceptionally in general (also in our experiments). In the reference sequence, $N$ 's do not appear alone but as chunks of $N$ 's. There are six chunks of $N$ 's in the reference and their lengths are $60,000,3,100,000,150,000,50,000,50,000$ and 50,000. In the positions where the reference sequence has N's, individual sequences also have N's mostly. In the other positions, most of N's in individual sequences are single N's. The chunks of N's in individual sequences may represent the regions that are not sequenced, the regions that are sequenced but have very low quality, or the regions that are moved to other places. Single N's in individual sequences represent positions where one character from $\{A, G, T, C\}$ cannot be determined

\footnotetext{
${ }^{6}$ http://samtools.sourceforge.net/
} 
Table 1. The number of non-common regions according to length.

\begin{tabular}{c|cccccc|c}
\hline Length & 1 & 2 & 3 & 4 & 5 & 6 & Total \\
\hline Number & 190,804 & 3,057 & 215 & 47 & 9 & 3 & 194,135 \\
\hline
\end{tabular}

Table 2. The lengths of $\alpha^{*}$ 's and $\alpha^{j}$ 's $(0 \leq j \leq 10)$.

\begin{tabular}{l|c|c||c|c|c}
\hline & Total length & Average length & & Total length & Average length \\
\hline$\alpha^{0}, \mathrm{~s}$ & $3,202,864$ & 16.50 & $\alpha^{6}, \mathrm{~s}$ & $2,987,607$ & 15.39 \\
$\alpha^{1}, \mathrm{~s}$ & $3,030,406$ & 15.61 & $\alpha^{7}, \mathrm{~s}$ & $3,022,359$ & 15.57 \\
$\alpha^{2}, \mathrm{~s}$ & $2,558,396$ & 13.18 & $\alpha^{8}, \mathrm{~s}$ & $3,132,487$ & 16.14 \\
$\alpha^{3}, \mathrm{~s}$ & $2,976,231$ & 15.33 & $\alpha^{9}, \mathrm{~s}$ & $3,026,544$ & 15.59 \\
$\alpha^{4}, \mathrm{~s}$ & $2,989,375$ & 15.40 & $\alpha^{10}, \mathrm{~s}$ & $3,140,456$ & 16.18 \\
\cline { 3 - 6 }$\alpha^{5}, \mathrm{~s}$ & $2,991,517$ & 15.14 & $\alpha^{*}, \mathrm{~s}$ & $3,229,589$ & 16.64 \\
\hline
\end{tabular}

because reads have different characters in $\{A, G, T, C\}$, there are deletions in reads, and/or the quality is low.

\subsection{Experimental results}

In this section, we compare the space requirements of the GSA and the SAA for the 11 sequences. For simplicity, we appended N's to the end of each individual sequence so that the length of the individual sequence is the same as that of the reference sequence. Let $S_{0}$ be the reference sequence and $S_{i}(1 \leq i \leq 10)$ be each individual sequence. We call an aligned position a non-common position if at least two distinct characters in $\{A, G, T, C\}$ appear at this position. Notice that we do not regard a position as a non-common position if $N$ and only one character in $\{A, G, T, C\}$ appear at the position. In our data set, there are $0.3 \%$ non-common positions (197,814 among 63,025,520 positions). Consecutive noncommon positions become a non-common region. There are 194,135 non-common regions whose lengths vary from 1 to 6 (Table 1 ).

We first compute the lengths of $\alpha^{*}$ 's in the sequences, which is a main factor for the space requirement of our index. For a common region $\alpha_{i}$, we denote by $\alpha_{i}^{j}$ the longest suffix of $\alpha_{i}$ appearing at least twice in sequence $S_{j}(0 \leq j \leq 10)$. Recall that $\alpha_{i}^{*}$ is the longest of $\alpha_{i}^{0}, \ldots, \alpha_{i}^{10}$. When computing $\alpha_{i}^{j}$, we exclude the part of $\alpha_{i}$ containing at least 10 consecutive $N$ 's since long consecutive $N$ 's do not carry any information about $\{A, G, T, C\}$. For each $j$, the total length and the average length of $\alpha_{i}^{j}$ 's are shown in Table 2. (We omit the subscript $i$ in $\alpha_{i}^{j}$ if not confusing.) For example, in sequence $S_{1}, 3,030,406$ characters $(4.8 \%)$ of the entire $63,025,520$ characters are included in $\alpha^{1}$ 's. Since there are 194,135 non-common regions, the average length of $\alpha^{1}$ 's is 15.61 .

From the lengths of non-common regions and $\alpha^{*}$ 's, we calculate the space requirement of the SAA. For a substring $\alpha \beta$ of a sequence $S_{j}$ where $\alpha$ is a common region and $\beta$ is a non-common region, we call $\alpha^{*} \beta$ a $N S$-region (nonshared region) and $\alpha^{\prime}$ a $S$-region (shared region) where $\alpha^{\prime}$ is the prefix of $\alpha$ such that $\alpha^{\prime} \alpha^{*}$ is $\alpha$. For a sequence, let $n_{t}$ be the length of the sequence, $n_{s}$ be the 
Table 3. Distribution of characters in our sequences.

\begin{tabular}{|c|c|c|c|c|}
\hline & \multicolumn{2}{|c|}{$\begin{array}{c}\text { NS-regions } \\
(3,427,403 \text { characters })\end{array}$} & \multicolumn{2}{|c|}{$\begin{array}{c}\text { S-regions } \\
(59,598,117 \text { characters })\end{array}$} \\
\hline & $\#$ of $\{A, G, T, C\}$ & $\#$ of $N$ & $\#$ of $\{A, G, T, C\}$ & \# of $N$ \\
\hline$S_{0}$ & $3,427,401$ & 2 & $56,078,119$ & $3,519,998$ \\
\hline$S_{1}$ & $3,318,768$ & 108,635 & $55,263,839$ & $4,334,278$ \\
\hline$S_{2}$ & $2,940,879$ & 486,524 & $49,719,051$ & $9,879,066$ \\
\hline$S_{3}$ & $3,272,652$ & 154,751 & $54,872,472$ & $4,725,645$ \\
\hline$S_{4}$ & $3,279,318$ & 148,085 & $54,969,949$ & $4,628,168$ \\
\hline$S_{5}$ & $3,285,414$ & 141,989 & $54,972,379$ & $4,625,738$ \\
\hline$S_{6}$ & $3,275,604$ & 151,799 & $54,947,026$ & $4,651,091$ \\
\hline$S_{7}$ & $3,306,405$ & 120,998 & $55,010,622$ & $4,587,495$ \\
\hline$S_{8}$ & $3,385,161$ & 42,242 & $55,717,092$ & $3,881,025$ \\
\hline$S_{9}$ & $3,311,346$ & 116,057 & $55,045,612$ & $4,552,505$ \\
\hline$S_{10}$ & $3,390,329$ & 37,074 & $55,722,788$ & $3,875,329$ \\
\hline Total & $36,193,277$ & $1,508,156$ & $602,318,949$ & $53,260,338$ \\
\hline
\end{tabular}

total length of S-regions, and $n_{n}$ be the total length of NS-regions. (Note that $n_{t}, n_{s}$, and $n_{n}$ are identical in all sequences and $n_{t}=n_{s}+n_{n}$.) Then, the size of the GSA is $11 n_{t}$ and the size of the SAA is $n_{s}+11 n_{n}\left(=n_{t}+10 n_{n}\right)$. In our data set, $n_{t}=63,025,520$ and $n_{n}=3,427,403$, and thus the size of the GSA is $693,280,720$ words and the size of the SAA is $97,299,550$ words. That is, our index uses only $14.03 \%$ space compared to the GSA.

When searching the sequences for a pattern, we may assume that the pattern does not contain $N$ since we do not consider wild-card matches. In this circumstance, we can reduce the space requirement of indexes by eliminating in indexes the suffixes whose first characters are $N$. To compute the sizes of the two indexes for our data set, we first investigate the distribution of $N$ in our sequences. Table 3 shows the distribution of characters in NS-regions and S-regions for each sequence. For example, in NS-regions of sequence $S_{1}$, the number of characters $A, G, T, C$ is $3,318,768(97 \%)$ and the number of character $N$ is $108,635(3 \%)$. In S-regions of sequence $S_{1}$, the number of characters $A, G, T, C$ is $55,263,839$ $(93 \%)$ and the number of character $N$ is $4,334,278(7 \%)$.

We compute the sizes of the two indexes when excluding the suffixes whose first characters are $N$. The size of the GSA is the total number of characters $A, G, T, C$ in NS-regions and S-regions of the 11 sequences, which is $638,512,226$ $(36,193,277+602,318,949)$ words (see Table 3$)$. Next, consider the SAA. For a position in an NS-region, we eliminate the suffix of each sequence starting at this position if the first character of the suffix is $N$. For a position in an S-region, we eliminate the suffix (a-suffix) starting at this position only if the characters in the position are $N$ in all sequences. In our data set, the total number of $A, G, T, C$ in NS-regions is 36,193,277 and the number of positions in S-regions excluding the positions where characters are $N$ in all sequences is $56,078,133$ (see the last row in Table 4). Thus, the size of our index is $92,271,410$ words, which is only $14.45 \%$ of the size of the GSA. 
Table 4. Comparison of the sizes of the GSA and the SAA according to the number of sequences when excluding the suffixes whose first characters are $N$. Column (C1) is the total number of $A, G, T, C$ in NS-regions and column (C2) is the number of positions in S-regions excluding the positions where characters are $N$ in all sequences. Then, the size of the SAA is $(\mathrm{C} 1)+(\mathrm{C} 2)$. The ratio of the size of the SAA to that of the GSA is given in the last column.

\begin{tabular}{c|cccc|c}
\hline & Size of GSA & Size of SAA & $(\mathrm{C} 1)$ & $(\mathrm{C} 2)$ & Ratio (\%) \\
\hline$S_{0} \sim S_{1}$ & $118,088,127$ & $60,455,692$ & $1,914,833$ & $58,540,859$ & 51.20 \\
$S_{0} \sim S_{2}$ & $170,748,057$ & $62,473,223$ & $4,553,672$ & $57,919,551$ & 36.59 \\
$S_{0} \sim S_{3}$ & $228,893,181$ & $65,338,136$ & $7,905,004$ & $57,433,132$ & 28.55 \\
$S_{0} \sim S_{4}$ & $287,142,448$ & $68,758,063$ & $11,706,204$ & $57,051,859$ & 23.95 \\
$S_{0} \sim S_{5}$ & $345,400,241$ & $72,483,002$ & $15,719,471$ & $56,763,531$ & 20.99 \\
$S_{0} \sim S_{6}$ & $403,622,871$ & $76,417,395$ & $19,881,933$ & $56,535,462$ & 18.93 \\
$S_{0} \sim S_{7}$ & $461,939,898$ & $80,305,819$ & $23,928,531$ & $56,377,288$ & 17.38 \\
$S_{0} \sim S_{8}$ & $521,042,151$ & $84,226,410$ & $27,963,745$ & $56,262,665$ & 16.16 \\
$S_{0} \sim S_{9}$ & $579,399,109$ & $88,223,461$ & $32,062,878$ & $56,160,583$ & 15.23 \\
$S_{0} \sim S_{10}$ & $638,512,226$ & $92,271,410$ & $36,193,277$ & $56,078,133$ & 14.45 \\
\hline
\end{tabular}

We also compare the space requirements of the GSA and the SAA according to the number of sequences used in indexing (Table 4). Obviously, the space efficiency of our index increases as the number of the sequences increases. The ratio of the space of the SAA to that of the GSA is $51.2 \%$ when two sequence are used, and the ratio is $14.45 \%$ when the 11 sequences are used.

\section{Acknowledgements}

Joong Chae Na was supported by Basic Science Research Program through the National Research Foundation of Korea(NRF) funded by the Ministry of Education, Science and Technology(2012-0003214), and by the IT R\&D program of MKE/KEIT [10038768, The Development of Supercomputing System for the Genome Analysis]. Heejin Park was supported by Basic Science Research Program through the National Research Foundation of Korea(NRF) funded by the Ministry of Education, Science and Technology(2012-0006999), by Seoul Creative Human Development Program (HM120006), by the Proteogenomics Research Program through the National Research Foundation of Korea funded by the Korean Ministry of Education, Science and Technology, and by the National Research Foundation of Korea(NRF) funded by the Ministry of Science, ICT \& Future Planning(2012-054452). Laurent Mouchard was supported by the French Ministry of Foreign Affairs Grant 27828RG (INDIGEN, PHC STAR 2012). Kunsoo Park was supported by National Research Foundation of Korea- Grant funded by the Korean Government(MSIP) (2012K1A3A4A07030483), and by Next-Generation Information Computing Development Program through the National Research Foundation of Korea(NRF) funded by the Ministry of Science, ICT \& Future Planning (2011-0029924). 


\section{References}

1. M. I. Abouelhoda, S. Kurtz, and E. Ohlebusch. Replacing suffix trees with enhanced suffix arrays. Journal of Discrete Algorithms, 2(1):53-86, 2004.

2. A. Apostolico. The myriad virtues of subword trees. In A. Apostolico and Z. Galil, editors, Combinatorial Algorithms on Words, pages 85-95. Springer, 1985.

3. M. Burrows and D. J. Wheeler. A block-sorting lossless data compression algorithm. Technical Report 124, Digital Equipment Corporation, Paolo Alto, California, 1994.

4. The 1000 Genomes Project Consortium. A map of human genome variation from population-scale sequencing. Nature, 467(7319):1061-1073, 2010.

5. H. H. Do, J. Jansson, K. Sadakane, and W. K. Sung. Fast relative Lempel-Ziv selfindex for similar sequences. In Proceedings of FAW-AAIM 2012, pages 291-302, 2012.

6. P. Ferragina and G. Manzini. Indexing compressed text. Journal of the ACM, 52(4):552-581, 2005.

7. R. Grossi and J. S. Vitter. Compressed suffix arrays and suffix trees with applications to text indexing and string matching. SIAM Journal on Computing, 35(2):378-407, 2005.

8. D. Gusfield. Algorithms on Strings, Tree, and Sequences. Cambridge University Press, Cambridge, 1997.

9. S. Huang, T. W. Lam, W. K. Sung, S. L. Tam, and S. M. Yiu. Indexing similar dna sequences. In Proceedings of AAIM 2010, pages 180-190, 2010.

10. J. Kärkkäinen, P. Sanders, and S. Burkhardt. Linear work suffix array construction. Journal of the ACM, 53(6):918-936, 2006.

11. D. K. Kim, M. Kim, and H. Park. Linearized suffix tree: an efficient index data structure with the capabilities of suffix trees and suffix arrays. Algorithmica, 52(3):350-377, 2008.

12. D. K. Kim, J. S. Sim, H. Park, and K. Park. Constructing suffix arrays in linear time. Journal of Discrete Algorithms, 3(2-4):126-142, 2005.

13. P. Ko and S. Aluru. Space efficient linear time construction of suffix arrays. Journal of Discrete Algorithms, 3(2-4):143-156, 2005.

14. S. Kreft and G. Navarro. On compressing and indexing repetitive sequences. Theoretical Computer Science, 483:115-133, 2013.

15. S. Kuruppu, S. J. Puglisi, and J. Zobel. Relative Lempel-Ziv compression of genomes for large-scale storage and retrieval. In Proceedings of SPIRE 2010, pages 201-206, 2010.

16. V. Mäkinen, G. Navarro, J. Sirén, and N. Välimäki. Storage and retrieval of individual genomes. In Proceedings of RECOMB 2009, pages 121-137, 2009.

17. V. Mäkinen, G. Navarro, J. Sirén, and N. Välimäki. Storage and retrieval of highly repetitive sequence collections. Journal of Computational Biology, 17(3):281-308, 2010.

18. U. Manber and G. Myers. Suffix arrays: A new method for on-line string searches. SIAM Journal on Computing, 22(5):935-948, 1993.

19. J. C. Na, M. Crochemore H. Park, J. Holub, C. S. Iliopoulos, L. Mouchard, and K. Park. Suffix tree of alignment: An efficient index for similar data. In Proceedings of IWOCA 2013, 2013.

20. G. Navarro. Indexing highly repetitive collections. In Proceedings of IWOCA 2012, pages 274-279, 2012.

21. J. Ziv and A. Lempel. A universal algorithm for sequential data compression. IEEE Transactions on Information Theory, 23(3):337-343, 1977. 